\title{
10 Sexualität, abweichendes Sozialverhalten und Geschlechterrollen delinquenter weiblicher Jugendlicher
}

Elena G. Dozortseva, Dimitry Davydov, Elena Morozova, Irina Savelieva, Iryna Sokolova, Svetlana Terekhina und Nikolai Vostroknutov

In den letzten Jahren stieg der Bedarf nach neuen differenzierten diagnostischen Ansätzen zur Erfassung der Probleme von jugendlichen Straftätern. Das hohe Tempo der Kriminalisierung von weiblichen Jugendlichen ist eine neue Erscheinung, die zurzeit die Jugendkriminalität in Russland charakterisiert. Dieses Phänomen verlangt weitere wissenschaftliche Untersuchungen und die Einrichtung innovativer Präventions- und Behandlungsmethoden, die die geschlechtsbedingten Besonderheiten dieser Klientinnen berücksichtigen. Einer der wichtigsten Bereiche der gesamten Persönlichkeitsformung bei diesen delinquenten weiblichen Jugendlichen ist die Förderung der psychosozialen und sexuellen Entwicklung.

Ein besonders gefährdender Aspekt besteht in der drastischen Zunahme von Geschlechtskrankheiten und HIV-Infektion bei diesen weiblichen Jugendlichen. Im Laufe der letzten Jahrzehnte stieg die Erkrankungshäufigkeit von Syphilis um das 7o-fache und die HIV-Erkrankungen hatten fast den Status einer Epidemie bekommen. Delinquente weibliche Jugendliche gehören zu einer besonderen Risikogruppe. Da die oben genannten Krankheiten von dem Sexualverhalten abhängen und deswegen als Verhaltensstörungen anzusehen 
10 Sexualität, abweichendes Sozialverhalten und Geschlechterrollen delinquenter weiblicher Jugendlicher

sind, spielen psychologische und psychiatrische Interventionen bei ihrer Behandlung und Prävention eine entscheidende Rolle.

Durch die bisher geringen klinischen Erfahrungen und wenigen wissenschaftlichen Erkenntnisse bedingt, besteht die Notwendigkeit von wissenschaftlichen Untersuchungen des psychosozialen und sexuellen Verhaltens der delinquenten weiblichen Jugendlichen, um ihre spezifischen Probleme zu identifizieren und entsprechende Interventionsmöglichkeiten für sie zu entwickeln. Das Ziel dieser Maßnahmen besteht in der Wiederherstellung ihrer „psychosozialen Stabilität und sexuellen Gesundheit“.

Unter Gesundheit wird nach der WHO-Definition ein „physischer, emotioneller und sozialer Zustand des Wohlbefindens" verstanden. Deswegen schließt der Begriff der „psychosexuellen Gesundheit“ medizinische (somatische und psychiatrische), psychologische und sozialpädagogische Aspekte ein. Da es um Jugendliche in Entwicklung geht, muss die psychosexuelle Integrität aus der Entwicklungsperspektive betrachtet werden und die altersgemäße somatische und psychische Reifung sowie die normale und altersgemäße Persönlichkeitsentwicklung als Referenz herangezogen werden.

Auf Basis dieses Konzepts wurde in Russland durch die Autorin umfangreiche multidisziplinäre Forschung durchgeführt. Die beteiligten Fachleute waren Psychologen, Psychiater und Gynäkologen. Im Rahmen dieses Forschungsprojektes wurden insgesamt go delinquente weibliche Jugendliche im Alter von 14 bis 17 Jahren in zwei spezifischen sozialpädagogischen Einrichtungen untersucht. Die Kontrollgruppe wurde aus 55 Schülerinnen im gleichen Alter im Bereich der Stadt Moskau gebildet.

Folgendes Untersuchungsdesign wurde verwandt:

1. Erfassung somatischer Basiswerte (Körperlänge, Körpergewicht, Brustumfang, Beinlänge usw.).Erfassung und Analyse der medizinischen Dokumentation unter besonderer Berücksichtigung der Erhebung einer Anamnese zum sexuellen und gynäkologischen Entwicklungsstand.

2. Erfassung und Analyse der medizinischen Dokumentation und der psychiatrischen Anamnese. Weiter wurde eine psychiatrische Diagnostik zur Erfassung von psychischen Störungen, Entwicklungsstörungen und Störungen im Sexualbereich durchgeführt.

3. Es wurde der Fragebogen „Selbsteinstellung“ von V. Stolin und Y. Pantileev sowie der Fragebogen zur Einstellung von Jugendlichen gegenüber ihren Eltern von V. Schaefer vorgelegt. Weiterhin wurden projektive Tests durchgeführt (Mensch-Zeichen-Test, Hand-Test und Rosenzweig Picture Frustration Test), Selbstbeurteilungsverfahren, Wahlmethoden (Terminalwerteliste von M. Rokich) durchgeführt, ein Farbassoziationstest vorgelegt und ein Interview zur psychologischen Untersuchung von Einstellungen, Werten und Verhaltensweisen bei den delinquenten Weibliche Jugendliche erhoben. 
10 Sexualität, abweichendes Sozialverhalten und Geschlechterrollen

delinquenter weiblicher Jugendlicher

Die nachfolgend dargestellten Ergebnisse kennzeichnen die gesamte Gruppe delinquenter weiblicher Jugendlicher.

Im somatischen Bereich wurde festgestellt, dass die Entwicklung des Knochenskeletts nach dem Frauentyp bei delinquenten weiblichen Jugendlichen früher und schneller verläuft und abgeschlossen war, als gemäß der Altersnorm zu erwarten gewesen wäre. Der Unterschied zeigt einen früheren Entwicklungsabschluss, der ca. zwei bis drei Jahre der normalen Entwicklung vorauseilt und dieses Phänomen ist besonders bei den jüngeren weiblichen Jugendlichen stark ausgeprägt. Es ist davon auszugehen, dass diese Erscheinung auf die Wirkung der Geschlechtshormone zurückzuführen ist. Insgesamt bleiben die delinquenten sozial auffälligen weiblichen Jugendlichen im Größenwachstum kleiner und erreichen nur ein geringeres Körpergewicht als die Gleichaltrigen aus der Kontrollgruppe.

Die Untersuchung der sexuellen Reife bei den delinquenten weiblichen Jugendlichen zeigte, dass das Durchschnittsalter des Eintritts der Menarche bei 12 Jahren lag. Das Durchschnittsalter des ersten sexuellen Kontakts der delinquenten weibliche jugendliche lag in dieser Cruppe bei 13,6 Jahren im Vergleich zu 15,6 Jahren im russischen Durchschnitt (16,4 Jahre bei Schülerinnen). Die angegebene Durchschnittszahl von Sexualpartnern lag bei 5 ( 1 bei den Schülerinnen) und der Prozentansatz von sexuell aktiven Jugendlichen unter den delinquenten weiblichen Jugendlichen war mit 6o,5\% höher ausgeprägt als bei gleichaltrigen Schülern mit 9,1\%.

45,2\% der weiblichen Jugendlichen dieser Gruppe hatten sich mit einer Geschlechtskrankheit vorher oder in der Zeit der Untersuchungen für die Studie infiziert. Dabei wurde deutlich, dass sie weitgehend keine vor Infektion schützenden oder andere Verhütungsmittel benutzten. 3\% von ihnen wurden unerwünscht schwanger und einige hatten schon vor Eintritt in die Studie einen Abbruch der Schwangerschaft durchgeführt. Dass es gegenüber der Erwartung der Autoren insgesamt dennoch eine geringere Anzahl von Schwangerschaften auftrat und es in der Folge wenig durchgeführte Interruptiones gab, wird darauf zurückgeführt, dass die Periode der sexuellen Aktivität der weiblichen Jugendlichen relativ kurz war und dass gleichzeitig eine hohe Morbidität an Geschlechtskrankheiten bei diesen weibliche Jugendlichen bestand, die ein Hindernis für die Entwicklung einer Schwangerschaft darstellten.

Nach psychiatrischer Untersuchung wurden 33,3\% der delinquenten weiblichen Jugendlichen als psychisch gesund kategorisiert, wobei jedoch Verwahrlosung, dissoziale Tendenzen, Störungen der Persönlichkeitsentwicklung, charakterliche Besonderheiten bzw. Akzentuierungen und episodischer Alkoholkonsum nicht als psychiatrisch auffällig bewertet wurden. In 31,2\% der Fälle wurden Anomalien der Persönlichkeitsentwicklung (Symptome pathologischer Persönlichkeitsentwicklung, Ausbildung einer Persönlichkeitsstörung) festgestellt. 41,9\% der weiblichen Jugendlichen erhielten die Diagnose einer Entwicklungsstörung unterschiedlicher Ausprägung bis zu schwerer 
10 Sexualität, abweichendes Sozialverhalten und Geschlechterrollen delinquenter weiblicher Jugendlicher

Entwicklungsretardation. Leichte kognitive Störungen waren in 30,1\% der Fälle festzustellen, darunter 23,6\% organisch bedingt. 22,6\% der Jugendlichen wiesen eine Substanzabhängigkeit auf (Alkohol, Drogen, Medikamente, Lösungsmittel). Bei 3,2\% der weiblichen Jugendlichen wurden Störungen der Geschlechtsidentität diagnostiziert und eine stabile homosexuelle Orientierung festgestellt. Unter den Persönlichkeitsabweichungen von verschiedenen Typen und Graden wurden am häufigsten „willenlose“, „haltlose“, histrionische und sensible Ausprägungsgrade gefunden.

Die psychopathologischen Befunde zeigten sich in folgenden zentralen Bereichen der psychosexuellen Entwicklung bei den delinquenten weiblichen Jugendlichen:

- Unreife Geschlechtsrollenidentität, unzureichende Identifizierung mit dem eigenen Geschlecht

- Abweichende Haltung gegenüber dem anderen Geschlecht

- Störungen der Entwicklung von Werten und Einstellungen in Bezug auf die Verhaltensweisen zwischen den Geschlechtern in verschiedenen Formen einschließlich der Familie und das reproduktive Verhalten

- Reduzierte Fähigkeit, sexuelle Impulse und sexuelles Verhalten altersangemessen und eigenständig bewusst zu steuern

Der Prozess der Entwicklung von Geschlechterrollen stellt ein zentrales Element zur Ausbildung einer integrierten Identität dar. Große Bedeutung hat dabei die emotionale Identifikation der weiblichen Jugendlichen mit den bedeutsamen weiblichen Bezugspersonen, vor allem mit der eigenen Mutter.

Die vorwiegende Identifizierung von delinquenten weiblichen Jugendlichen mit dem weiblichen Geschlecht zeigt sich in projektiven Verfahren beim Zeichnen von Menschenfiguren. Die Zeichnungen der delinquenten weiblichen Jugendlichen stellen viel häufiger weibliche Jugendliche und Frauenfiguren dar, als dies in der Kontrollgruppe beobachtet wurde, jeweils 85,5\% und 62,9\% bei $<<0$,o1 ein signifikanter Unterschied in der Häufigkeit der Darstellungen. Auch die Ergebnisse des sogenannten „psycho-semantischen Farbassoziationstests“(eine Methode, die die subjektive Realität und die inneren semantischen Zusammenhänge von Probanden widerspiegeln soll) weisen auf die emotionale Nähe von „Ich-Vorstellungen“ und Begriffen „Frau“ und „Weibliche Jugendliche" hin.

Dennoch zeigten sich einige Hindernisse, die den Integrationsprozess für die dissozialen bzw. delinquenten weiblichen Jugendlichen erschweren. Ein Problem ist, dass Identität und Autonomie bei den delinquenten weiblichen Jugendliche kaum entwickelt zu sein scheint. Es fehlten ihnen eine realistische Selbstwahrnehmung und ein rollenspezifisches Selbstverständnis. Dem unklaren und unbeständigen aktuellen „Ich“ steht das positiv erlebte ideale „erwünschte Ich“ und das „künftige Ich“ gegenüber, die beide eine „unproduktive“ und „pseudokompensatorische“ Rolle spielen. Statt wirkliche innere 
10 Sexualität, abweichendes Sozialverhalten und Geschlechterrollen delinquenter weiblicher Jugendlicher

Veränderungen und Entwicklungen zu realisieren, verharren die weiblichen Jugendlichen in ihren Phantasien in Form von Wunschbildern einer ,idealen Zukunft".

Ein weiteres Problem ist durch die intrafamiliäre Beziehungsstörung bedingt, vor allem durch die belastete Beziehung zwischen den weiblichen Jugendlichen und ihren Müttern. Die Ergebnisse zeigten (s. Abb. 4), dass das positive Interesse und die Autonomie die Haupttendenzen in den Einstellungen von den Müttern zu ihren Töchtern der Kontrollgruppe sind. In der Gruppe der delinquenten weiblichen Jugendlichen stehen dagegen Feindseligkeit, Unbeständigkeit und direktive Verhaltensweisen im Vordergrund. Solche Haltungen verhindern eine positive emotionale Identifikation der Töchter mit ihren Müttern sowie die Norm entsprechende soziale Vermittlung von Frauenrollen und einer angemessenen und entwicklungsförderlichen sozialen Position in der Familie. Es wurde weiter festgestellt, dass im Unterschied zu den nicht-delinquenten weiblichen Jugendlichen die Selbsteinstellung von delinquenten weiblichen Jugendlichen sehr hohe Korrelationen mit den Einstellungen der Mutter aufwiesen, so dass das Selbsterleben und Verhalten dieser weiblichen Jugendlichen oft eine kritiklose und unbewusste Übernahme der Haltungen der Mütter ist. Dies bestätigen andere Befunde, die Unselbständigkeit und Mangel an eigener positiver Identität bei den weiblichen Jugendlichen zeigen.

Die Entwicklung der Wahrnehmung des anderen Geschlechts sowie die Einstellung zu Männern werden bei dem weiblichen Jugendlichen in der Normgruppe von der „Vaterfigur“ geprägt. Die „Vaterfigur“ beeinflusste wesentlich die Vorstellungen der Töchter über ihren möglichen späteren Partner bzw.

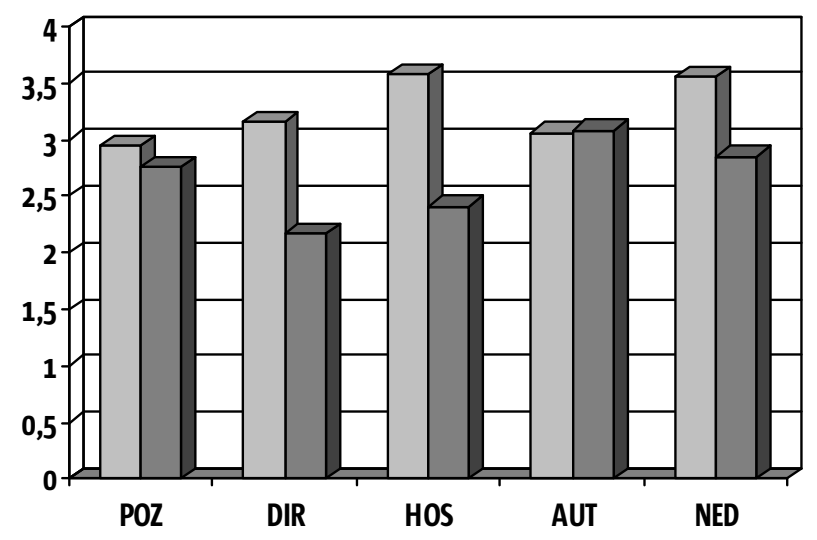

$\square$ Delinq. $\quad \square$ Kontroll.

Abb. 4 Wahrnehmung des Verhaltens der Mutter bei delinquenten und nicht-delinquenten weiblichen Jugendlichen (ADOR-Test) 
10 Sexualität, abweichendes Sozialverhalten und Geschlechterrollen delinquenter weiblicher Jugendlicher

Ehemann. Bei den delinquenten weiblichen Jugendlichen zeigte sich dies beeinträchtigt. Nur die Hälfte der weiblichen Jugendlichen kannte ihren Vater, und selbst wenn er in der Familie präsent war, diente er selten in irgendeiner Form als soziales Vorbild. Ca. 53,7\% dieser Väter waren alkoholabhängig und $21,4 \%$ in verschiedener Weise straffällig geworden. Die Einstellungen der Väter zu den Töchtern ähnelten denen der Mütter. Die weiblichen Jugendlichen assoziieren in ihren Vorstellungen ihre Väter mit den Begriffen „Schwäche“ und „Gleichgültigkeit“ (Farbassoziationstest). Dies zeigt, dass der Vater für die delinquenten weiblichen Jugendlichen kein relevanter Rollenträger oder ein Vorbild war, der eine männliche Geschlechterrolle positiv repräsentieren und vermitteln könnte. Die Vorstellungen der betroffenen weiblichen Jugendlichen von Ihren zukünftigen Partnern/Ehemännern sind somit eher Projektionen der eigenen idealen Ich-Vorstellungen, die kaum einen Bezug zur Realität aufweisen.

Die zukünftige Familie erschien für die delinquenten weiblichen Jugendlichen von großer subjektiver Bedeutung (s. Abb. 5). In der Liste von 19 Terminalwerten haben $57,9 \%$ der weiblichen Jugendlichen Familie als eine der drei wichtigen „Lebenswerte“ genannt (29,7\% in der Kontrollgruppe, $\mathrm{p}<0,01)$. Das Problem zeigt sich darin, dass die Familienbildung nicht als eine wichtige soziale und persönliche Aufgabe erlebt wird, sondern als eine „magische“ Lösung der bestehenden emotionalen und sozialen Probleme. Auf diese Weise spielen die Vorstellungen von der zukünftigen Familie zusätzlich eine kompensatorische Rolle. Die weiblichen Jugendlichen der Cruppe streben nach baldiger Erfüllung dieser Träume: 68,9\% planten bzw. wünschten die Eheschließung vor dem 22. Lebensjahr, während $57 \%$ der Schülerinnen aus der Kontrollgruppe an eine

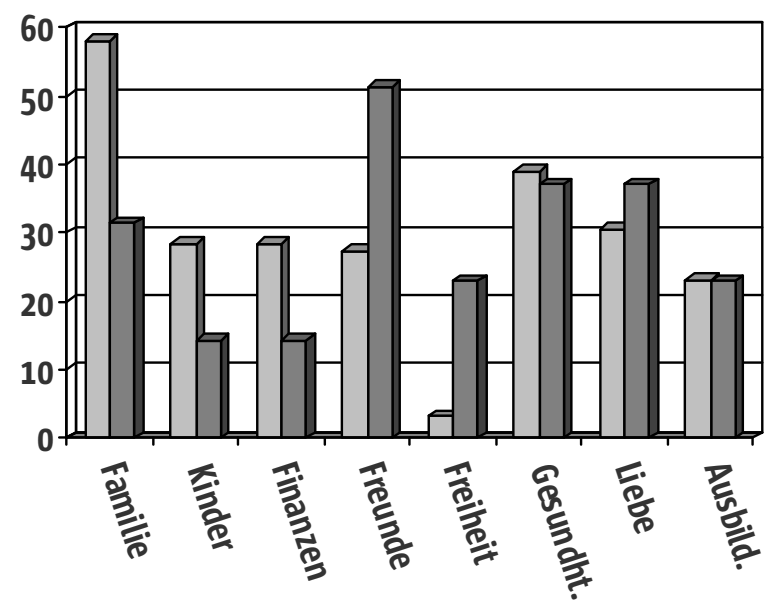

Delinq. $\quad \square$ Kontroll.

Abb. 5 Werte bei delinquenten und nicht-delinquenten weiblichen Jugendlichen in \% 
10 Sexualität, abweichendes Sozialverhalten und Geschlechterrollen

Ehe erst deutlich nach diesem Alter denken. Dies lässt den Schluss zu, dass die delinquenten weiblichen Jugendlichen für das Familienleben unvorbereitet bleiben und in der unmittelbaren Zukunft Enttäuschungen erleben können.

Die Einstellung der weiblichen Jugendlichen zur Sexualität unterschied sich deutlich zwischen der delinquenten weiblichen Jugendlichengruppe und der Kontrollgruppe. Im subjektiven inneren Bild der delinquenten weiblichen Jugendlichen wird der Begriff Sex mit „Geld“ assoziiert, was vermutlich mit ihren frühen Erfahrungen in diesem Bereich in Zusammenhang steht, dass finanzielle Aspekte mit der Sexualität verbunden sind.

Die Daten in jedem Untersuchungsbereich (Psychologie, Psychiatrie, Gynäkologie und Sexologie) offenbaren wichtige Aspekte, die hier einzeln dargestellt werden müssen. Die gemeinsame Analyse dieser Befunde erlaubt, neue Sichtweisen der Probleme und eine weitere Differenzierung zwischen den Cruppen.

Es wurde festgestellt, dass die schon oben dargestellte Neigung, in der eigenen Familie eine „emotionale Herberge“ $z u$ suchen, nicht für alle weiblichen Jugendlichen typisch ist, sondern meist nur diejenigen charakterisiert, die wegen der frühkindlichen Entwicklungsstörung eine defizitäre Entwicklung mit Zeichen mangelnder kognitiver Leistungsfähigkeit oder kognitiver Störungen aufweisen. Die weiblichen Jugendlichen zeigen positive soziale Einstellungen, Bereitschaft zur Arbeit, positive (obwohl nicht immer kritische) Selbsteinschätzungen, jedoch auf der anderen Seite einen ausgesprochenen Mangel an Fähigkeiten, ihr Verhalten eigenständig zu steuern. Die delinquenten weiblichen Jugendlichen waren unreif, passiv, „willensschwach“, neigen zu Konformität und sie zeigten sich als stark von den Einschätzungen und Meinungen der unmittelbaren sozialen Umgebung abhängig. Sie stammten aus Familien, in denen ein dirigistischer, autoritärer und rigider Erziehungsstil vorherrschte und sie wiesen eine gewisse Bereitschaft auf, sich wiederum unter gleichartige Kontrollbedingungen zu stellen.

Die Delinquenz trat meistens im Rahmen von devianten gemischtgeschlechtlichen Gruppen auf und wurde durch diese Cruppen unterstützt und initiiert.

In den pädagogischen Einrichtungen neigen die delinquenten weiblichen Jugendlichen sehr schnell dazu sich anzupassen, sie verhalten sich unauffällig und werden aus diesem Crunde als „gut erziehbar“ eingeschätzt. Dennoch hing die Kriminalprognose in großem Umfang von dem sozialen Rahmen ab, in dem sie leben bzw. in den sie nach einer Intervention sie integriert werden können. Die delinquenten weiblichen Jugendlichen, die in entsprechende pädagogische oder therapeutische Behandlungseinrichtungen integriert worden waren, zeigten weniger sexuelle Aktivität und litten auch weniger unter den negativen psychosozialen Folgen von früher altersunangemessener sexueller Aktivität.

Die Behandlung mit diesen weiblichen Jugendlichen sollte vor allem geeigneten pädagogisch und psychotherapeutisch ausgerichteten Einrichtungen er- 
10 Sexualität, abweichendes Sozialverhalten und Geschlechterrollen delinquenter weiblicher Jugendlicher

folgen. Das Ziel der Intervention liegt idealerweise in der Stärkung von Selbständigkeit, Reflexionsfähigkeit sowie in der Förderung aktiver, autonomer und sozial orientierter Verhaltenssteuerung, um die Kompetenzen für eine bewusste Verhaltenssteuerung zu fördern und die Persönlichkeitsentwicklung im allgemeinen zu stützen.

Die psychiatrische Begleitung ist in den meisten Fällen notwendig, um die Folgen der psychiatrischen und medizinisch-organischen Störungen zu behandeln und Behinderungen und Defizite so weit möglich zu kompensieren sowie den körperlich-funktionellen Zustand stabil zu erhalten. Weiterhin dienen die multidisziplinären bzw. multimodalen Behandlungsansätze dazu, die Betroffenen angemessenen am Arbeitsplatz und im sozialen Raum zu integrieren, damit sie die dort gestellten Erwartungen an Leistung und sozialer Kompetenz im Team auf der Basis ihrer weiter entwickelten Fähigkeiten erfüllen können. Die sozialpädagogische und psychiatrische bzw. psychotherapeutische Betreuung richtet sich an den folgenden Schwerpunkten aus:

- Sexualpädagogik,

- Entwicklung von Werten im interpersonalen Bereich,

- Entwicklung von Geschlechterrollen und Familienrollenbildern,

- Stärkung der Kompetenz bei der Bewältigung von Familienproblemen einschließlich Partnerschaftsbeziehungen und Kindererziehung.

Dies soll dazu dienen, die weiblichen Jugendlichen auf ein zukünftiges, sozial adäquates, kompetentes Leben in der sozialen Gemeinschaft und in einer Familie vorzubereiten.

Die andere Gruppe der delinquenten weiblichen Jugendlichen ist durch eine vielfältig und schwer gestörte Entwicklung charakterisiert, die aus einem Symptomenkomplex von äußerst schwerwiegenden psychologischen, psychiatrischen Beeinträchtigungen und sexuellen Auffälligkeiten besteht. Die weiblichen Jugendlichen sind vor allem durch Störungen in der Persönlichkeitsentwicklung gekennzeichnet und zeigen hauptsächlich Merkmale einer Persönlichkeitsstörung vom impulsiven, haltlosen oder/und histrionischen Typ bei durchschnittlicher intellektueller Leistungsfähigkeit. Sie zeigen ein hohes Maß an plan- und sinnloser Aktivität, an Impulsivität, sie weisen starke innere Konflikte mit negativen Selbstbewertungen auf und zeigen Erwartungen und Einschätzungen von anderen Menschen, die ihrem eigenen Selbstbild entsprechen. Weiterhin ist ihr Verhalten durch Fremd- und bzw. oder Autoaggressivität gekennzeichnet, in vielen Fällen zeigen sie ausgeprägte dissoziale Tendenzen, die hauptsächlich durch Alkohol- und Drogenkonsum sowie ein unstrukturiertes und selbstgefährdendes Sexualverhalten charakterisiert und belastet werden.

Die Analyse der Sexualanamnese lässt den Schluss zu, dass die Erfahrungen in diesem Bereich massive beeinträchtigende Auswirkungen auf die Persönlichkeitsentwicklung der weiblichen Jugendlichen haben. Es ist nicht auszuschließen, dass Besonderheiten und Abweichungen der Wirkung weiblicher Hormo- 
10 Sexualität, abweichendes Sozialverhalten und Geschlechterrollen delinquenter weiblicher Jugendlicher

ne sowie sozialen Verhaltensstörungen diesen Prozess fördern bzw. ursächlich bedingen. Je früher die negativen oder beeinträchtigenden sexuellen Erfahrungen gemacht wurden, desto größer war die Wahrscheinlichkeit, dass sexuelle Gewalt gegen diese weiblichen Jugendlichen ausgeübt wurde. 46,3\% der jungen Frauen hatten ihre ersten sexuellen Erfahrungen mit 13 Jahren und früher, 82,9\% mit 14 Jahren und früher. Unter den weiblichen Jugendlichen, die ihre ersten sexuellen Kontakte mit 12 Jahren oder davor hatten, sind 85,7\% vergewaltigt worden (s. Abb. 6). Im Alter von 13 Jahren sind 58\%, im Alter von 14 Jahren 50\% durch sexuelle Gewalt, vornehmlich Vergewaltigungen, belastet. Über das Alter von 15 Jahren wurden keine Vergewaltigungen dokumentiert. Diese Vergewaltigungen wurden meist von erwachsenen Männern begangen. 57,9\% der ersten sexuellen Kontakte mit einem erwachsenen Partner hatten Gewaltcharakter, dabei wurde in 22,2\% der Fälle die Gewalt bei den sexuellen Übergriffen durch Gleichaltrige ausgeübt. Die Bandbreite und Ausprägungsformen der negativen Einflüsse der sexuellen Gewalt auf den psychischen Zustand und auf die Persönlichkeitsentwicklung der weiblichen Jugendlichen sind bekannt. Die Daten dieser Untersuchung bestätigen diese Befunde. Weiter zeigten sich bei den Vergewaltigungsopfern veränderte eigene Wertschätzung und Selbstwertgefühl, abweichende Einstellungen zu anderen Personen und zu wichtigen Werten der Gemeinschaft. Die weiblichen Jugendlichen lehnen ,Liebe“ als Wert ab, schätzen eine positive und optimistische Einstellung im Umgang mit der Gegenwart und Erwartung für die Zukunft niedrig ein und wollen nicht sozial angepasstes und prosoziales Verhalten zeigen. Sie erwarten kein positives Verhalten von ihrer Umgebung und erleben ausgeprägte Schuldgefühle.

Die weibliche Jugendliche mit frühen sexuellen Erfahrungen zeigen später häufig Partnerwechsel und eine erhöhte Inzidenz von Geschlechtskrankheiten. Die

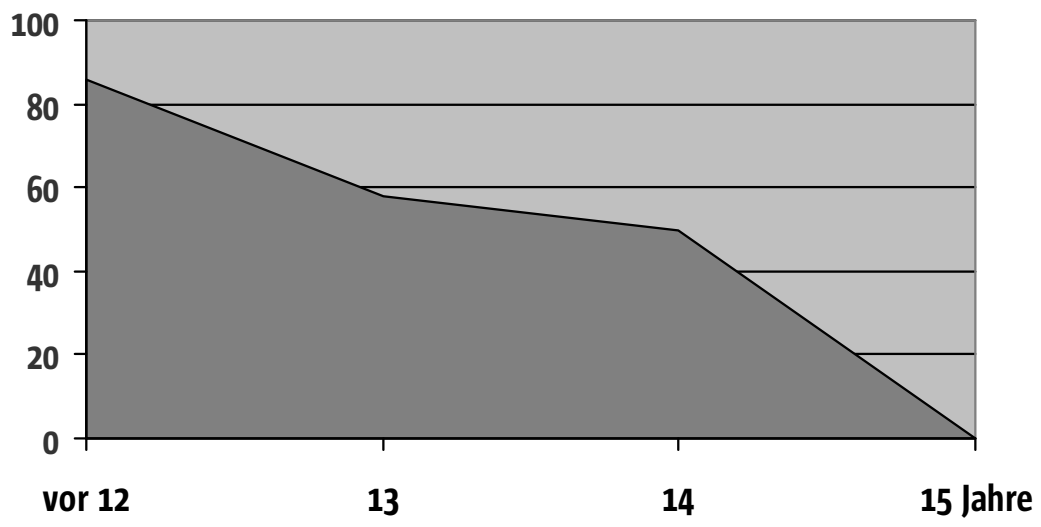

$\square$ Prozentsatz von Vergewaltigungen

Abb. 6 Vergewaltigung als erster Sexualkontakt bei delinquenten weiblichen Jugendlichen $(\mathrm{N}=49)$ 
10 Sexualität, abweichendes Sozialverhalten und Geschlechterrollen delinquenter weiblicher Jugendlicher

frühen sexuellen Erfahrungen haben nicht nur somatische Auswirkungen, sondern sie beeinträchtigen auch den psychischen Zustand der weiblichen Jugendlichen, sie erhöhen die Ausprägung von Ängstlichkeit, Erregbarkeit und Aggressivität, und die Wahrscheinlichkeit für das Erleben von Minderwertigkeitsgefühlen. Der Begriff „Sex“ bekommt für diese weiblichen Jugendlichen eine negative emotionale Färbung und wird subjektiv mit „Schmerz“ und Trauma assoziiert.

Die weiblichen Jugendlichen mit Kennzeichen in Sinne von Vorerfahrungen im Rahmen eines sogenannten „sexuellen Komplex“ (frühe Geschlechtskontakte, Vergewaltigung, häufige Partnerwechsel, Geschlechtskrankheiten) verschieben die Möglichkeit, eine Beziehung einzugehen, eine Ehe zu schließen in die weite Zukunft, bis zum Alter von 28 bis 30 Jahren. Viele von ihnen möchten zwar ein Kind, aber ohne Ehe oder Partnerschaft haben. Das Bedürfnis nach und die hohe Bewertung von Mutterschaft ist besonders bei den weibliche Jugendlichen ausgeprägt, die an Geschlechtskrankheiten leiden und daran zweifeln, ob sie zukünftig jemals schwanger werden könnten.

Die frühen sexuellen Erfahrungen zeigten eine hohe Korrelation mit Drogenkonsum, dessen negative Auswirkungen auf den psychischen Zustand und das Wertesystem als bekannt vorausgesetzt werden kann. Im Fall des Drogenkonsums kann bei den delinquenten weiblichen Jugendlichen die Neigung zur Ausbildung einer negativen Identität beobachtet werden.

Die Behandlung der weiblichen Jugendlichen mit einer Vielzahl ineinander verflochtener Störungen, Defizite und Verhaltensprobleme bedingt einen komplexen sozialpädagogischen und therapeutischen Ansatz. Der Anfang der Behandlung beginnt mit medizinisch-somatischen Maßnahmen, um die körperlichen Störungen (z.B. sexuell übertragene Infektionen) zu behandeln, die die körperliche Integrität der weiblichen Jugendlichen deutlich beeinträchtigen und deren Behandlung deshalb erste Präferenz hat. Weiterhin findet eine medizinisch-pädagogische Aufklärung und Begleitung im Bereich der Sexualität statt, die in erster Linie von Gynäkologen durchgeführt wird. Diese Beratung wird durch die Beteiligung von Psychologen ergänzt, um durch psychologisch therapeutische Stützung eine Auseinandersetzung mit und die Entwicklung von sozialen Normvorstellungen und ethischen Werten zu ermöglichen. Hinzu kommt die Bearbeitung die individuellen Probleme der Betroffenen. Auch wenn die Fragen und Sorgen der weiblichen Jugendlichen zuerst anonym in der Gruppe besprochen werden könnten, schaffen die in einer vertraulichen Atmosphäre durchgeführten individuellen pädagogisch-psychologischen Beratungen eine vertrauensvolle Beziehung. Diese Atmosphäre erlaubt, die Problembesprechung mit den weiblichen Jugendlichen einzeln fortzusetzen. Wenn die weiblichen Jugendlichen erleben, dass sie mit ihren intimen Problemen akzeptiert und ernst genommen werden, führt das zu einer „Normalisierung“ ihrer Selbstwahrnehmung und hilft ihnen, ihr Selbstbild positiv zu verändern. Dies schafft die weitere Basis für die 
Einzel- und Gruppenarbeit mit dem Ziel, ausreichende Coping-Strategien, Fähigkeiten zu Selbstkritik und Selbstreflexion sowie für eine bewusste Verhaltenssteuerung zu entwickeln. Gleichzeitig ist eine psychotherapeutischpsychiatrische Behandlung des aus den erlebten sexuellen Gewalthandlungen herrührenden Traumas durchzuführen. Die therapeutische Arbeit mit den drogenabhängigen weiblichen Jugendliche und jungen Frauen sollte ein Bestandteil dieses Prozesses werden. Wichtig ist, eigene Ressourcen der weiblichen Jugendliche zu identifizieren und zu fördern: Positive soziale Motive, emotionale Bindungen, realistische Zukunftsplanung. Der für das spätere Leben geplante Kinderwunsch kann ein Anknüpfungspunkt in der Therapie sein. Familiäre Rollen und partnerschaftliche Beziehungen müssen in diesem Zusammenhang ausführlich bearbeitet und entwickelt werden.

Mit einer Zunahme der Anzahl delinquenter und sexuell auffälliger weiblicher Jugendlicher wird in Russland gerechnet und es besteht die Absicht und der Wille, die bestehende Hilfesysteme in Vorbereitung für diese Entwicklung auszubauen.

\section{Literatur}

Dozortseva EG (2005) Psychic Trauma in Juvenile Delinquents: Gender Specifics Serbsky National Center for Social and Forensic Psychiatry. Moscow, Russia, XXIX International Congress on Law \& Mental Health.

Dozortseva EG (2005) The Best Interest of the Child: The Russian View Serbsky Center for Social and Forensic Psychiatry. Moscow, Russia, XXIXth International Congress on Law \& Mental Health.

Dozortseva EG, Loginova EV (2007) Psychological Characteristics of Adolescent Gang Offences. Psychological Science and Education 5: 289-294.

Dozortseva EG, Malantseva OD (2010) Psychological Characteristics of Adolescents Prone to Xenophobia. Psychological Science and Education 2: 44-53.

Kachaeva MA, Dozortseva EG, Davydov DM (2000) System-structural analysis for aggressive criminal behavior in females with psychiatric disorders. Russ | Psychiatry 3: 33-6.

Koudrjavtsev JA, Dozortseva EG (1988) Psychological age: Theory and legal expertise. Psikologicheskii Zhurnal 9(6): 103-115. 